In reality, it is highly unlikely that there has been a true rise and fall in homicide among mentally ill people in England and Wales over the past 50 years. These figures are entirely based on statistics which reflect the workings of the Criminal Justice system (a charge to which I plead guilty). ${ }^{2}$ They merely reflect changes in processing defendants by the courts. The probable culprit for declining diminished responsibility was declining enthusiasm for treating personality disordered and sexually deviant killers under the Mental Health Act legal category 'Psychopathic Disorder'. The authors did not provide statistics on other forms of manslaughter. These have increased in recent years, suggesting that defence lawyers have become more successful in putting forward alternative defences to murder than diminished responsibility.

I agree with the authors that sociological and legal factors (mainly the latter) have effects on rates of homicide due to mental disorder. But it is the overall base rate of homicide in the population that matters and with which these figures must be compared. This differs markedly between different countries. In those where it is very high, such as South America and Sub-Saharan Africa, mental disorder is almost irrelevant as an epidemiological risk factor. The authors refer to a small number of studies suggesting a correlation between rates of homicide among the mentally ill and rates among the rest of the population. It may well be that the "laws' ${ }^{2}$ they refer to are too rigid. For example, it makes sense that a country that allows handgun ownership is more likely to have killers with schizophrenia who use a handgun, and at a rate higher than in countries where handguns are banned, although the evidence for this remains thin on the ground. But from the public health perspective does it matter? Handguns are the key risk factor, not schizophrenia.

England and Wales have a low but steadily rising rate of homicide. It is unrealistic to propose mental health services as a public health intervention, but will be popular with politicians. Social geographers have demonstrated that social exclusion and growing social inequalities are the strongest correlates with this phenomenon affecting young men in England and Wales. ${ }^{3}$

1 Large M, Smith G, Swinson N, Shaw J, Nielson O. Homicide due to mental disorder in England and Wales over 50 years. Br J Psychiatry 2008; 193: 130-3.

2 Coid J. The epidemiology of abnormal homicide and murder followed by suicide. Psychol Med 1983; 13: 855-60.

3 Shaw $M$, Tunstall $H$, Dorling $D$. Increasing inequalities in risk of murder in Britain. Trends in the demographic and spatial distribution of murder, 1981-2000. Health Place 2005; 11: 45-54.

Jeremy w. Coid, St Bartholomew's Hospital, 61 Bartholomew Close, London EC1A 7BE, UK. Email: j.w.coid@qmul.ac.uk

doi: 10.1192/bjp.194.2.185b

Authors' reply: We welcome interest in our study of homicide in England and Wales. However, we disagree with Coid's assertion that the conclusions are illogical because the same social factors that were associated with the increase in homicides by the mentally ill up to the 1970s were present when those homicides declined. There are several possible reasons for decline in homicide by the mentally ill, including the availability of treatment. Coid's assertion that a fall in homicide due to better treatment must mean that the earlier rise was due to deteriorating mental health services is a similar oversimplification.

There has been no change in the law regarding diminished responsibility since 1957. Coid's explanation that the decline in homicide by the mentally ill since the late 1970s was due to a change in the threshold for the verdict of diminished responsibility is not supported by any data. Moreover, a change in threshold for diminished responsibility would not explain the decline in the verdicts of 'not guilty due to mental illness', 'permanently unfit for trial' and 'infanticide'. We also defend the use of legal outcomes to define cases. Given the careful attention paid to homicide matters by the courts, their verdicts are likely to be reasonably sensitive and highly specific. ${ }^{1}$

Vinkers et al report 8 years of data from The Netherlands, without showing that rates of homicide by the mentally ill have declined over a longer period. However, a lack of a decline in The Netherlands might not be unexpected, as we have found that $40 \%$ of homicides in psychotic illness occur before treatment, ${ }^{1}$ that delay in the initial treatment of schizophrenia is associated with a greater proportion of homicides during the first episode of psychosis $^{2}$ and that jurisdictions with mental health laws that require a patient to be dangerous before they can receive involuntary psychiatric treatment, such as The Netherlands, have longer delays in the treatment of early psychosis. ${ }^{3}$

We look forward to a challenge to our findings based on data rather than opinion and speculation.

1 Nielssen O, Large M. Rates of homicide during the first episode of psychosis and after treatment: a systematic review and meta-analysis. Schizophr Bull 2008; doi: 10.1093/schbul/sbn144 (Epub ahead of print).

2 Large $\mathrm{M}$, Nielssen $\mathrm{O}$. Evidence for a relationship between the duration of untreated psychosis and the proportion of psychotic homicides prior to treatment. Soc Psychiatry Psychiatr Epidemiol 2008; 43: 37-44.

3 Large M, Nielssen O, Ryan C, Hayes R. Mental health laws that require dangerousness for involuntary treatment may delay the initial treatment of schizophrenia. Soc Psychiatry Psychiatr Epidemiol 2008; 43: 251-6.

Matthew Large, Mental Health Services, St Vincent's Hospital, 299 Forbes Street, Darlinghurst, Sydney, NSW 2010, Australia. Email: mmbl@bigpond.com; Glen Smith, Northern Sydney Central Coast Area Health Service, Macquarie Hospital, North Ryde, New South Wales, Australia; Olav Nielssen, Mental Health Services, St Vincent's Hospital, and Clinical Research Unit for Anxiety Disorders, School of Psychiatry, UNSW at St Vincent's Hospital, Darlinghurst, Sydney, New South Wales, Australia

doi: 10.1192/bjp.194.2.186

\section{Antipsychotics and risk of diabetes in schizophrenia}

Smith et al state that there is increasing concern among clinicians about the association between second-generation antipsychotics and diabetes. ${ }^{1}$

It is interesting then that while commenting on the lack of systematic reviews and meta-analyses that support this concern, the authors go on to investigate not the relationship between starting antipsychotics and developing diabetes, but the relative risk of developing diabetes between groups of patients commenced on first-generation and second-generation antipsychotics. It is questionable whether this meta-analysis addresses, in any clinically meaningful way, the risk of developing diabetes after starting an antipsychotic, whether second or first generation. This would appear to be more usefully addressed by looking at the absolute risk.

The authors report on the difficulties in finding high-quality trials to include in their study. This is illustrated by the inclusion of only 11 trials out of an identified 1974. Smith et al then go on to outline their own criteria for a study to be considered of 'high quality'. These criteria include a prospective design and at least 1 year of follow-up recorded. It is of note then that of the 11 studies eventually included in the analysis, only 3 were prospective. Furthermore, of these 3 prospective trials, none was longer than 3 months. All trials included in the review could, therefore, be classified as low quality. The test for heterogeneity between studies, applied by the authors, further illustrates the highly significant methodological heterogeneity between studies. 